\title{
Nucleon form factors in dispersively improved chiral effective field theory. II. Electromagnetic form factors
}

\author{
J. M. Alarcón* and C. Weiss ${ }^{\dagger}$ \\ Theory Center, Jefferson Lab, Newport News, Virginia 23606, USA
}

(Received 19 October 2017; revised manuscript received 17 January 2018; published 8 May 2018)

\begin{abstract}
We study the nucleon electromagnetic form factors (EM FFs) using a recently developed method combining chiral effective field theory ( $\chi$ EFT) and dispersion analysis. The spectral functions on the two-pion cut at $t>4 M_{\pi}^{2}$ are constructed using the elastic unitarity relation and an $N / D$ representation. $\chi$ EFT is used to calculate the real functions $J_{ \pm}^{1}(t)=f_{ \pm}^{1}(t) / F_{\pi}(t)$ (ratios of the complex $\pi \pi \rightarrow N \bar{N}$ partial-wave amplitudes and the timelike pion $\mathrm{FF})$, which are free of $\pi \pi$ rescattering. Rescattering effects are included through the empirical timelike pion $\mathrm{FF}\left|F_{\pi}(t)\right|^{2}$. The method allows us to compute the isovector EM spectral functions up to $t \sim 1 \mathrm{GeV}^{2}$ with controlled accuracy (leading order, next-to-leading order, and partial next-to-next-to-leading order). With the spectral functions we calculate the isovector nucleon EM FFs and their derivatives at $t=0$ (EM radii, moments) using subtracted dispersion relations. We predict the values of higher FF derivatives, which are not affected by higher-order chiral corrections and are obtained almost parameter-free in our approach, and explain their collective behavior. We estimate the individual proton and neutron FFs by adding an empirical parametrization of the isoscalar sector. Excellent agreement with the present low- $Q^{2} \mathrm{FF}$ data is achieved up to $\sim 0.5 \mathrm{GeV}^{2}$ for $G_{E}$, and up to $\sim 0.2 \mathrm{GeV}^{2}$ for $G_{M}$. Our results can be used to guide the analysis of low- $Q^{2}$ elastic scattering data and the extraction of the proton charge radius.
\end{abstract}

DOI: 10.1103/PhysRevC.97.055203

\section{INTRODUCTION}

The electromagnetic form factors (EM FFs) describe the nucleon's elastic response to external EM fields and reveal the spatial distribution of charge and magnetization inside the strongly interacting system. They are among the most basic characteristics of nucleon structure and have been the object of extensive theoretical and experimental study. The electric and magnetic FFs at invariant momentum transfers $t<0$ are measured in elastic electron-nucleon scattering. Experiments at $|t| \leqslant 1 \mathrm{GeV}^{2}$ have been performed at many facilities, most recently at the Mainz Microtron (MAMI) [1-3] and at Jefferson Lab (JLab) [4-6]; see Refs. [7-9] for a review of the other data. The derivative of the proton electric FF at $t=0$, or charge radius, governs the nucleon structure corrections to the energy levels of hydrogen atoms (electronic or muonic) and is measured with high precision in atomic physics experiments. Recent experimental results have raised interesting questions regarding the precise value of the proton charge radius and the extrapolation of the elastic scattering data to $t \rightarrow 0$; see Refs. [10-12] for a review. Theoretical calculations of the

\footnotetext{
*alarcon@jlab.org

${ }^{\dagger}$ weiss@jlab.org
}

Published by the American Physical Society under the terms of the Creative Commons Attribution 4.0 International license. Further distribution of this work must maintain attribution to the author $(s)$ and the published article's title, journal citation, and DOI. Funded by $S C O A P^{3}$. nucleon FFs at $|t| \ll 1 \mathrm{GeV}^{2}$ are needed to guide the analysis of the experimental data and help answer these questions. Dedicated measurements of the proton electric FF at extremely low momentum transfers $|t| \geqslant 10^{-4} \mathrm{GeV}^{2}$ are planned at JLab [13]. Knowledge of the low- $t$ EM FFs is also required for constructing the peripheral transverse densities and generalized parton distributions (GPDs) of the nucleon $[14,15]$.

In a previous article we proposed a method for calculating the nucleon FFs of $G$-parity-even operators by combining chiral effective field theory ( $\chi$ EFT) and dispersion analysis (dispersively improved $\chi \mathrm{EFT}$, or DI $\chi \mathrm{EFT}$ ) [16]. It starts from the dispersive representation of the FFs as analytic functions of $t$ and constructs the spectral functions on the two-pion cut at $t>4 M_{\pi}^{2}$ using the elastic unitarity relation [17,18]. An $N / D$ representation is employed to separate the coupling of the $\pi \pi$ system to the nucleon from the effects of $\pi \pi$ rescattering in the $t$ channel. $\chi$ EFT is used to calculate the real functions $J(t)=$ $f(t) / F_{\pi}(t)$ : the ratios of the complex $\pi \pi \rightarrow N \bar{N}$ partial-wave amplitudes (PWAs) and the timelike pion FF, which are free of $\pi \pi$ rescattering and show good convergence. Rescattering effects are included through the timelike pion $\mathrm{FF}\left|F_{\pi}(t)\right|^{2}$, which is taken from sources outside of $\chi$ EFT [experimental data, lattice QCD (LQCD)]. The formulation permits firstprinciples calculations of the two-pion spectral functions of the FFs with controlled accuracy. The spectral functions can then be used to evaluate the FFs and related quantities of interest (nucleon radii, transverse densities) with subtracted dispersion relations. The method results in a dramatic improvement compared to conventional $\chi \mathrm{EFT}$ calculations of the spectral functions of nucleon FFs [19-23]. The $\pi \pi$ rescattering effects included through $\left|F_{\pi}(t)\right|^{2}$ substantially increase the $\chi \mathrm{EFT}$ 
results in the near-threshold region $t-4 M_{\pi}^{2} \sim$ few $M_{\pi}^{2}$ and allow one to extend the spectral function calculations into the region of the $\pi \pi$ resonances at $t \lesssim 1 \mathrm{GeV}^{2}$; see Refs. [15,16] for details. In Ref. [16] the method was applied to the nucleon isoscalar-scalar FF, where the $\pi \pi$ system in the $t$ channel is in the $I=J=0$ state. The resulting spectral functions and FFs were found to be in good agreement with those of empirical dispersion theory and Roy-Steiner equations [24,25].

In the present article we use the DI $\chi$ EFT method to calculate the nucleon EM FFs at low $t$ and study their properties. We construct the isovector EM spectral functions on the two-pion cut by combining the elastic unitarity relation in the $I=J=1$ channel, the $N / D$ representation; $\chi$ EFT calculations of the $J$ functions at leading order (LO), next-to-leading order (NLO), and partial next-to-next-to-leading order (N2LO) accuracy; and the timelike pion $\mathrm{FF}\left|F_{\pi}\right|^{2}$ measured in $e^{+} e^{-}$annihilation experiments. Realistic spectral functions are obtained up to $t \sim 1 \mathrm{GeV}^{2}$, which includes the $\rho$ meson region essential for EM structure. With these spectral functions we evaluate the isovector FFs and their derivatives (radii) using subtracted dispersion relations. We obtain the individual proton and neutron FFs by supplementing the calculated isovector spectral functions with an empirical parametrization of the isoscalar ones [15]. Excellent agreement with the low- $t$ EM FF data is achieved.

In particular, the method allows us to predict the higher derivatives of the EM FFs at $t=0$ and explain their collective behavior. They are given by well-convergent dispersion integrals, which can be evaluated reliably with the DI $\chi$ EFT spectral functions, with minimal model dependence. The higher derivatives are governed by two disparate dynamical scales-the vector meson mass, $M_{V}^{2}(V=\rho, \omega)$, and the twopion threshold, $4 M_{\pi}^{2}$ - and exhibit a rich structure. The values of the higher derivatives therefore differ qualitatively from what one would estimate based on a single dynamical scale (naturalness). Recent attempts to fit the low- $t$ proton electric FF data and extract the charge radius have engendered a debate regarding the values of higher FF derivatives and their role in the $t \rightarrow 0$ extrapolation [1,2,26-31]. Our predictions for the higher derivatives can be compared with those obtained in form factor fits (regarding order-of-magnitude, collective behavior) and used to discriminate between different fits. Our method incorporates the exact analytic structure of the FF in $t$ and the multiple dynamical scales governing its behavior, which are essential in the analysis of low- $t$ FF data and the extraction of charge radius.

The plan of the article is as follows. In Sec. II we describe the steps of the DI $\chi$ EFT calculation, expanding on the general description of the method in Ref. [16] and emphasizing the aspects that are new or specific to the EM FFs. This includes the unitarity relations and $N / D$ representation in the $I=J=1$ channel, the LO $\chi$ EFT calculation of the $J$ functions, the estimate of higher-order corrections, and the parametrization of the timelike pion FF. In Sec. III we present the results and their interpretation. This covers the isovector nucleon EM spectral functions, the nucleon EM radii, higher derivatives (moments) of the EM FFs and their structure, and the spacelike nucleon FFs at low $|t|$. In Sec. IV we summarize the results and comment on possible further applications of the method.
A similar method for calculating nucleon FFs, combining $\chi$ EFT and dispersion theory, was described in Ref. [32] and applied to the EM FFs in Ref. [33]. The differences from our approach are mainly in the technical implementation of the $\pi \pi$ rescattering effects in the unitarity relations $(N / D$ method vs Omnes function) and the estimates of higher-order chiral corrections. Nucleon FFs were also studied in an extension of $\chi$ EFT with explicit vector meson degrees of freedom in Ref. [34]. The low $-t$ nucleon FFs and their derivatives were also calculated in heavy-baryon $\chi$ EFT with $\Delta$ 's, in the context of a study of two-photon exchange corrections to muonic hydrogen in Ref. [35].

\section{CALCUlation}

\section{A. Nucleon EM form factors in DI $\chi$ EFT}

The transition matrix element of the EM current between nucleon (proton, neutron) states is parametrized by the invariant FFs $F_{1}(t)$ and $F_{2}(t)$ (Dirac and Pauli FFs; we use the conventions of Ref. [15]). The electric and magnetic FFs are defined as

$$
\begin{aligned}
G_{E}(t) & =F_{1}(t)-\tau F_{2}(t), \\
G_{M}(t) & =F_{1}(t)+F_{2}(t),
\end{aligned}
$$

where $\tau \equiv-t /\left(4 m_{N}^{2}\right)$. At zero momentum transfer the electric FF gives the electric charge of the nucleon, $G_{E}^{p, n}(0)=(1,0)$, and the magnetic FF gives the total (pointlike plus anomalous) magnetic moment in units of nuclear magnetons, $G_{M}^{p, n}(0)=$ $\mu^{p, n}=(2.79,-1.91)$. The isovector and isoscalar components are defined as

$$
G_{i}^{V, S} \equiv \frac{1}{2}\left(G_{i}^{p} \mp G_{i}^{n}\right) \quad(i=E, M) .
$$

The FFs are analytic functions of $t$ and satisfy dispersion relations. They express the FFs at complex $t$ as an integral over their singularities at real $t>0$, corresponding to (unphysical) timelike processes in which the current couples to the nucleon through exchange of a hadronic system in the $t$ channel. In the case of the isovector EM FFs the lowest-mass hadronic state is the $\pi \pi$ state, and the dispersion integrals start at $t^{\prime}=4 M_{\pi}^{2}$ (two-pion threshold):

$$
G_{i}^{V}(t)=\frac{1}{\pi} \int_{4 M_{\pi}^{2}}^{\infty} d t^{\prime} \frac{\operatorname{Im} G_{i}^{V}\left(t^{\prime}\right)}{t^{\prime}-t-i 0} \quad(i=E, M) .
$$

The real functions $\operatorname{Im} G_{i}^{V}\left(t^{\prime}\right)\left(t^{\prime}>4 M_{\pi}^{2}\right)$ are referred to as the spectral functions. At $4 M_{\pi}^{2}<t^{\prime}<16 M_{\pi}^{2}$ the $\pi \pi$ state is the only possible state contributing to $\operatorname{Im} G_{i}^{V}\left(t^{\prime}\right)$. It is known from $e^{+} e^{-}$annihilation experiments that higher states ( $4 \pi$ etc.) do not couple strongly to the EM current, and it is expected that the $\pi \pi$ channel in the nucleon spectral functions remains dominant up to $t^{\prime} \sim 1 \mathrm{GeV}^{2}$. In this region the $\pi \pi$ channel includes the $\rho$ resonance at $t^{\prime}=M_{\rho}^{2} \sim 0.6 \mathrm{GeV}^{2}$, which has a decisive influence on the EM FFs. In the isoscalar case the dispersion integral starts with the $3 \pi$ state at $t^{\prime}=9 M_{\pi}^{2}$, with the dominant strength at $t<1 \mathrm{GeV}^{2}$ coming from the $\omega$ resonance at $t^{\prime}=$ $M_{\omega}^{2} \sim 0.6 \mathrm{GeV}^{2}$. The contribution of higher-mass states to the isovector and isoscalar FFs is constrained by the total charge and magnetic moment and has been determined empirically through fits of spacelike FF data [36,37]; the exact composition 
of these multihadron states is poorly known and will not be needed in the following applications.

In the region $4 M_{\pi}^{2}<t<16 M_{\pi}^{2}$ the isovector spectral functions on the two-pion cut can be obtained from the elastic unitarity relations $[17,18]$

$$
\begin{aligned}
& \operatorname{Im} G_{E}^{V}(t)=\frac{k_{\mathrm{cm}}^{3}}{m_{N} \sqrt{t}} f_{+}^{1}(t) F_{\pi}^{*}(t), \\
& \operatorname{Im} G_{M}^{V}(t)=\frac{k_{\mathrm{cm}}^{3}}{\sqrt{2 t}} f_{-}^{1}(t) F_{\pi}^{*}(t),
\end{aligned}
$$

where $k_{\mathrm{cm}} \equiv \sqrt{t / 4-M_{\pi}^{2}}$ is the center-of-mass momentum of the $\pi \pi$ system in the $t$ channel, $f_{ \pm}^{1}(t)$ are the $\pi \pi \rightarrow N \bar{N}$ PWAs in the normalization of Ref. [36], and $F_{\pi}^{*}(t)$ is the complex-conjugate timelike pion EM FF. Equations (5) and (6) are valid strictly in the region up to the $4 \pi$ threshold, $4 M_{\pi}^{2}<t<16 M_{\pi}^{2}$; if contributions from $4 \pi$ and higher states are neglected they can effectively be used up to $t \sim 1 \mathrm{GeV}^{2}=$ $50 M_{\pi}^{2}$. The expressions on the right-hand side of Eqs. (5) and (6) are real because the complex functions $f_{ \pm}^{1}(t)$ and $F_{\pi}(t)$ have the same phase on the two-pion cut (Watson theorem) [38]. The unitarity relations can therefore be written in a manifestly real form as $[17,18,39]$

$$
\begin{aligned}
\operatorname{Im} G_{E}^{V}(t) & =\frac{k_{\mathrm{cm}}^{3}}{m_{N} \sqrt{t}} J_{+}^{1}(t)\left|F_{\pi}(t)\right|^{2}, \\
\operatorname{Im} G_{M}^{V}(t) & =\frac{k_{\mathrm{cm}}^{3}}{\sqrt{2 t}} J_{-}^{1}(t)\left|F_{\pi}(t)\right|^{2},
\end{aligned}
$$

where

$$
J_{ \pm}^{1}(t) \equiv \frac{f_{ \pm}^{1}(t)}{F_{\pi}(t)}
$$

The functions $J_{ \pm}^{1}(t)$ are real for $t>4 M_{\pi}^{2}$ and thus have no right-hand cut; their only singularities are left-hand cuts at $t<$ $4 M_{\pi}^{2}-M_{\pi}^{4} / m_{N}^{2}$, the threshold resulting from the singularity of the nucleon Born term in the $\pi \pi \rightarrow N \bar{N}$ PWAs. Equations (7)-(9) are equivalent to a particular $N / D$ representation of the PWAs [40],

$$
f_{ \pm}^{1}(t)=\frac{J_{ \pm}^{1}(t)}{D(t)}, \quad D(t) \equiv 1 / F_{\pi}(t)
$$

in which the numerator functions $J_{ \pm}^{1}(t)$ contain the left-hand cut and the denominator function $1 / F_{\pi}(t)$ contains the righthand cut.

To evaluate the spectral functions in the representation of Eqs. (7)-(9), following Ref. [16], we calculate the real functions $J_{ \pm}^{1}(t)$ in $\chi$ EFT and multiply them with the empirical pion FF modulus $\left|F_{\pi}\right|^{2}$. Advantages of this approach are the following:

(a) The $\chi$ EFT calculation of $J_{ \pm}^{1}(t)$ is free of $\pi \pi$ rescattering and shows good convergence. Rescattering effects are entirely contained in $\left|F_{\pi}(t)\right|^{2}$, which is taken from other sources. In traditional "direct" $\chi$ EFT calculations of the spectral functions the $\pi \pi$ rescattering effects result in large higher-order corrections and render the perturbative expansion impractical.

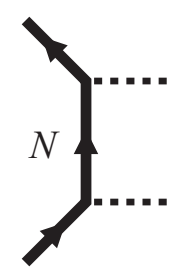

(a)

(d)

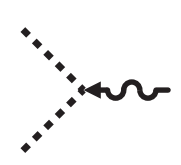

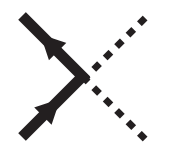

(b)

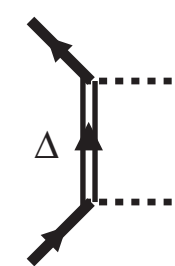

(c)
FIG. 1. (a)-(c) LO $\chi$ EFT diagrams contributing to the $\pi \pi \rightarrow$ $N \bar{N}$ PWA in the $I=J=1$ channel. (a) $N$ Born term. (b) WeinbergTomozawa contact term. (c) $\Delta$ Born term. (d) Pion EM FF in LO.

(b) The functions $J_{ \pm}^{1}(t)$ are dominated by the scales $M_{\pi}$ and $m_{\Delta}-m_{N}$ associated with the Born graph singularities, while $\left|F_{\pi}(t)\right|^{2}$ is dominated by the chiral-symmetrybreaking scale $\Lambda_{\chi}$. The representation Eqs. (7)-(9) is therefore consistent with the idea of separation of scales.

(c) The squared modulus $\left|F_{\pi}(t)\right|^{2}$ can be imported directly from the $e^{+} e^{-} \rightarrow \pi^{+} \pi^{-}$data or from LQCD calculations without determination of the phase [16,41].

For further discussion of the method we refer to Ref. [16].

\section{B. Leading-order calculation}

For calculating the $J$ functions of Eq. (9) we use $\chi$ EFT with the $\mathrm{SU}(2)$-flavor group and relativistic $N$ and $\Delta$ degrees of freedom. This formulation ensures the correct position of the singularities and includes the important contributions from the $\Delta$ Born term. The setup of the $\chi$ EFT calculation is described in Ref. [42] and summarized in Ref. [15] (fields, chiral Lagrangian, power counting, values of couplings). The interactions of the spin-3/2 $\Delta$ field are formulated with consistent vertices [43-46], and the extended-on-mass-shell (EOMS) scheme is used to maintain the standard power counting [47] (diagrams with pion loops do not enter in the present calculation).

The LO diagrams contributing to the $\pi \pi \rightarrow N \bar{N}$ PWAs in the $I=J=1$ channel are shown in Fig. 1. They include the $N$ and $\Delta$ Born terms, Figs. 1(a) and 1(c), and the WeinbergTomozawa contact term, Fig. 1(b), which appears as the result of chiral invariance of the dynamics with relativistic baryons. We take the results for the $\mathrm{LO} \pi N \rightarrow \pi N$ amplitude of Ref. [42] (the first relativistic $\chi \mathrm{EFT}$ calculation of $\pi N$ scattering with explicit $\Delta$ ), and project onto the $I=J=1$ channel to get the PWAs $f_{ \pm}^{1}(t)$. The pion EM FF at this order is just $F_{\pi}(t)=1$ (pointlike); see Fig. 1(d). The $\chi$ EFT results for $J_{ \pm}^{1}(t)$ therefore coincide with $f_{ \pm}^{1}(t)$ at this order. Analytic expressions for $J_{ \pm}^{1}(t)$ are given in Appendix A. Numerical results of the LO approximation will be shown below. 


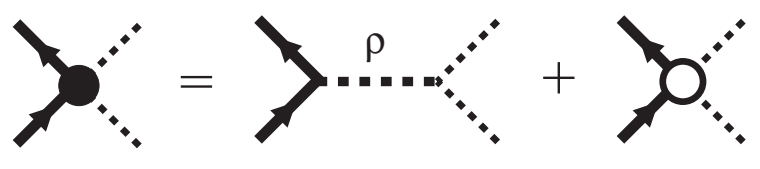

FIG. 2. Adjustment of the LEC of the NLO $\pi \pi N N$ contact term, $c_{4}$, in the DI $\chi$ EFT approach. The original contact term (filled circle, left-hand side) is equated with the sum of $\rho$ meson exchange and the adjusted contact term (open circle).

\section{Estimates of higher-order corrections}

At NLO accuracy, corrections to the $I=J=1 \pi \pi \rightarrow N \bar{N}$ PWAs arise from the NLO contact term in the chiral Lagrangian with the low-energy constant (LEC) $c_{4}$. The value of this LEC has to be adjusted consistently with our unitarity-based approach [15]. In standard $\chi$ EFT calculations $c_{4}$ receives large contribution from $\rho$ meson exchange. Since in our formulation the effect of the $\rho$ is included explicitly through $\left|F_{\pi}(t)\right|^{2}$, we have to remove it from the value of $c_{4}$ to avoid double counting (see Fig. 2). Using the estimate for the $\rho$ contribution of Ref. [48], $c_{4}^{\rho} \approx 1.63 \mathrm{GeV}^{-1}$, and subtracting it from the empirical $c_{4}$ reported in Refs. [42,49], we obtain the range

$$
c_{4} \text { [adjusted] }=(-0.54,0.27) \mathrm{GeV}^{-1} \text {. }
$$

Note that these values are much smaller than the original $c_{4}$ and consistent with zero, which means that the NLO corrections to the isovector spectral functions in our formulation are very small. The analytic expressions for the NLO corrections to $J_{ \pm}^{1}(t)$ are given in Appendix A.

At N2LO accuracy pion loop corrections appear, and the structure of the $\chi$ EFT expressions becomes considerably richer. The $\pi \pi \rightarrow N \bar{N}$ PWAs and the pion FF now involve $\pi \pi$ rescattering in the $t$ channel and become complex at $t>4 M_{\pi}^{2}$, in such a way that their phases cancel and the functions $J_{ \pm}^{1}(t)$ of Eq. (9) remain real. Also, $\pi N$ and $\pi \Delta s$-channel intermediate states appear in the $\pi N$ amplitude. Following Ref. [15] we perform a simple estimate of the N2LO corrections to the spectral functions of the electric FF, by taking the N2LO treelevel amplitudes and fixing the LECs through the charge sum rule. We require that the unsubtracted dispersion relation for the isovector electric FF reproduce the isovector charge when the integration is restricted to the region $t^{\prime}<t_{\max } \sim 1 \mathrm{GeV}^{2}$,

$$
\frac{1}{\pi} \int_{4 M_{\pi}^{2}}^{t_{\max }} d t^{\prime} \frac{\operatorname{Im} G_{E}^{V}\left(t^{\prime}\right)}{t^{\prime}}=\frac{1}{2} .
$$

This condition gives N2LO contact term contributions with sign opposite to that of the LO and NLO results, which provides a crucial curvature in the electric spectral function and allows us to extend the calculations up to $t \sim 1 \mathrm{GeV}^{2}$. In the language of traditional dispersion analysis these contact terms represent the negative contributions from the $\rho^{\prime}$, which compensate the excess charge that would be produced by the $\rho$ alone. We refer to this partial account of the N2LO corrections as pN2LO. In the magnetic FF no new tree-level amplitudes with LECs enter at N2LO level, so that the described method of estimating of the corrections cannot be applied. Our calculations of $G_{M}^{V}$ are therefore limited to NLO accuracy, and are expected to describe the empirical spectral functions only at $t \ll 1 \mathrm{GeV}^{2}$.

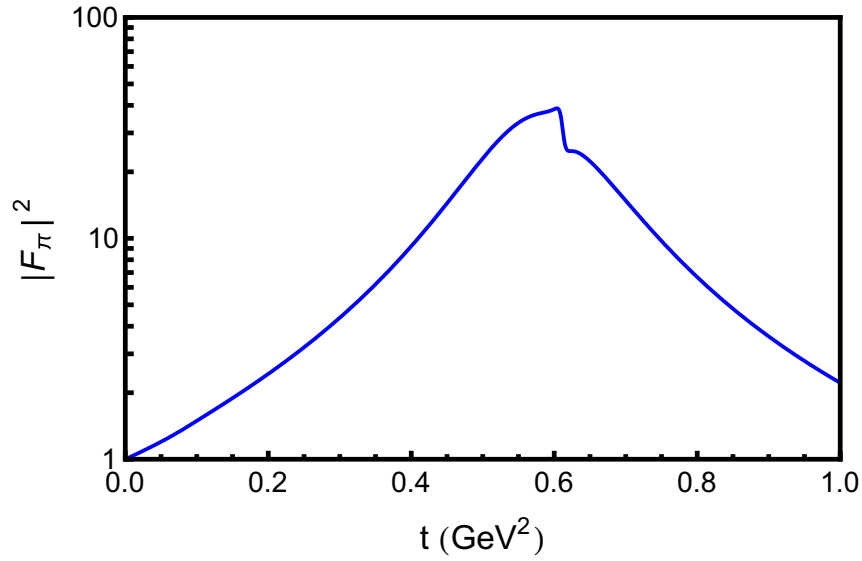

FIG. 3. Empirical parametrization of the timelike pion EM FF $\left|F_{\pi}(t)\right|^{2}$ obtained from $e^{+} e^{-} \rightarrow \pi^{+} \pi^{-}$annihilation data (details see text).

Numerical results of the NLO and pN2LO approximations will be shown below.

\section{Timelike pion EM form factor}

For evaluating the timelike pion FF entering in our calculation we use a Gounaris-Sakurai parametrization of the $e^{+} e^{-} \rightarrow$ $\pi^{+} \pi^{-}$exclusive annihilation data [50], including effects of $\rho-\omega$ mixing [51], with the parameters determined in Ref. [26]. The squared modulus $\left|F_{\pi}(t)\right|^{2}$ is shown in Fig. 3. One clearly sees the $\rho$ resonance at $t \sim 0.6 \mathrm{GeV}^{2}$ and the rapid variation resulting from $\rho-\omega$ mixing. The fact that $\left|F_{\pi}(t)\right|^{2}$ reaches a value of $\sim 2$ at $t \sim 0.2 \mathrm{GeV}^{2}$, and $\sim 10$ at $t \sim 0.4 \mathrm{GeV}^{2}$, shows that $\pi \pi$ rescattering is very substantial already at moderate $t$ and justifies our approach of incorporating these effects empirically.

Since $\left|F_{\pi}(t)\right|^{2}$ is determined very accurately from the annihilation data, we neglect the effect of its uncertainty on the spectral functions. In the following we quote only the uncertainties of the spectral function resulting from the $\chi \mathrm{EFT}$ calculation of $J_{ \pm}^{1}(t)$.

\section{RESULTS}

\section{A. Isovector EM spectral functions}

The spectral functions of the isovector EM nucleon FFs are evaluated using the DI $\chi$ EFT method and parameters described in Sec. II. Figure 4 shows the results of the $\chi$ EFT calculation of the real functions $J_{ \pm}^{1}(t)$ at $t>4 M_{\pi}^{2}$ [Eq. (9)]. For a better view the plot shows the functions multiplied by the kinematic factors of Eqs. (7) and (8), $k_{\mathrm{cm}}^{3} /\left(m_{N} \sqrt{t}\right)$ and $k_{\mathrm{cm}}^{3} / \sqrt{2 t}$, respectively. One observes the following:

(a) The $J_{ \pm}^{1}(t)$ with the kinematic factors are smooth functions, as expected on grounds of their analytic properties.

(b) The $\chi$ EFT calculations of $J_{ \pm}^{1}(t)$ show good convergence. In both functions higher-order corrections are small at threshold and increase with $t$. LO and NLO results are close because the adjusted LEC $c_{4}$ is small 

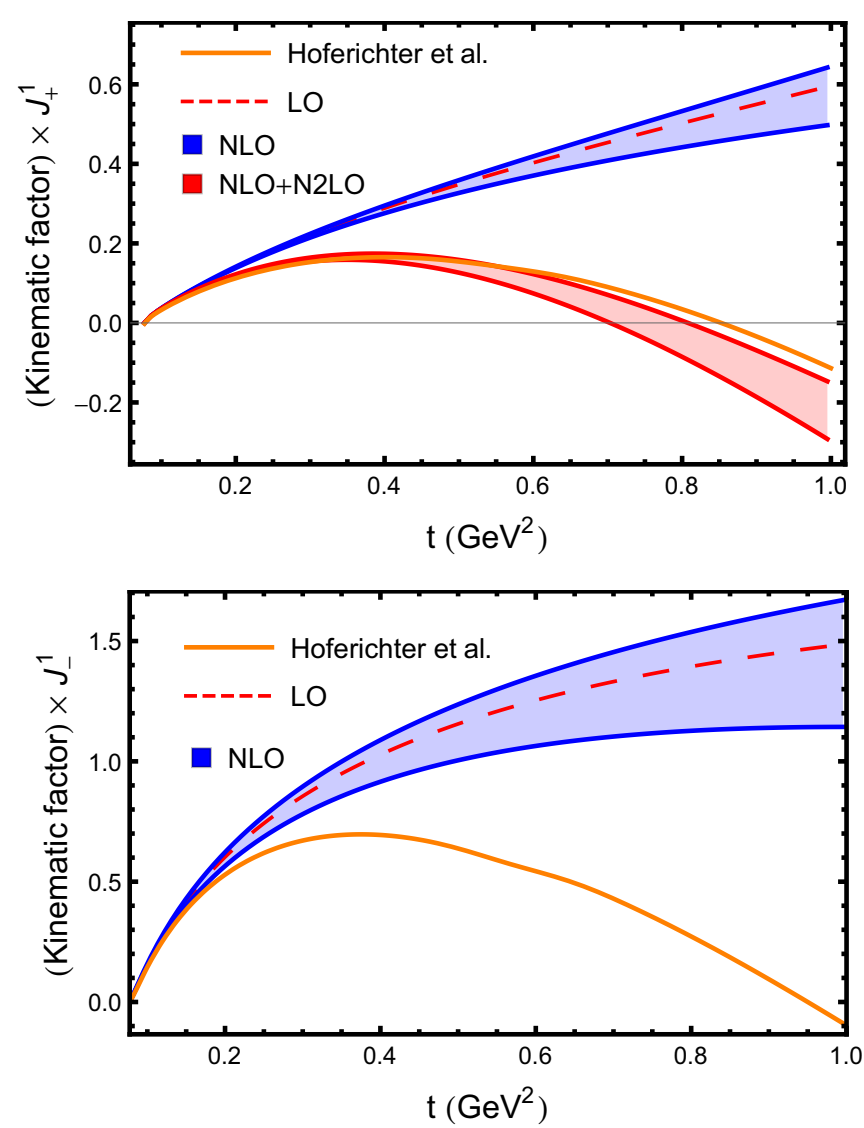

FIG. 4. $\chi$ EFT results for the functions $k_{\mathrm{cm}}^{3} /\left(m_{N} \sqrt{t}\right) J_{+}^{1}(t)$ and $k_{\mathrm{cm}}^{3} /(\sqrt{2 t}) J_{-}^{1}(t)$, Eqs.(7)-(9), at $t>4 M_{\pi}^{2}$. Dashed lines: LO approximation. Blue bands: NLO approximation. Red band: NLO+pN2LO, estimated as described in Sec. IIC. Solid orange lines: Roy-Steiner analysis results [52].

[Eq. (11)]. In $J_{+}^{1}(t)$ the pN2LO corrections, estimated as described in Sec. IIC, are negative and cause the function to decrease and turn negative at larger $t$.

(c) The $\chi$ EFT results for $J_{ \pm}^{1}(t)$ show reasonable agreement with the functions extracted from an analysis of $\pi N$ scattering data using Roy-Steiner equations [52]. In both $J_{+}^{1}$ and $J_{-}^{1}$ the LO and NLO approximation agree with the Roy-Steiner result up to $t \sim 0.2 \mathrm{GeV}^{2}$. In $J_{+}^{1}$ the negative $\mathrm{pN} 2 \mathrm{LO}$ corrections extend the region of agreement up to $t \sim 1 \mathrm{GeV}^{2}$. Note that the large change between NLO and pN2LO at $t>0.5 \mathrm{GeV}^{2}$ suggests that higher-order corrections beyond those included here might be important in this region.

Here and in the following, the error bands in the figures only show the uncertainty resulting from the variation of the LECs at a given order; the total uncertainty should be inferred taking into account also the discrepancy between the LO, NLO, and pN2LO approximations.

Figure 5 shows the isovector spectral functions, obtained by multiplying the $\chi$ EFT results for $J_{ \pm}^{1}(t)$ with the empirical $\left|F_{\pi}(t)\right|^{2}$, cf. Eqs. (7) and (8). The spectral functions clearly show the effects of $\pi \pi$ rescattering, which are not suitable
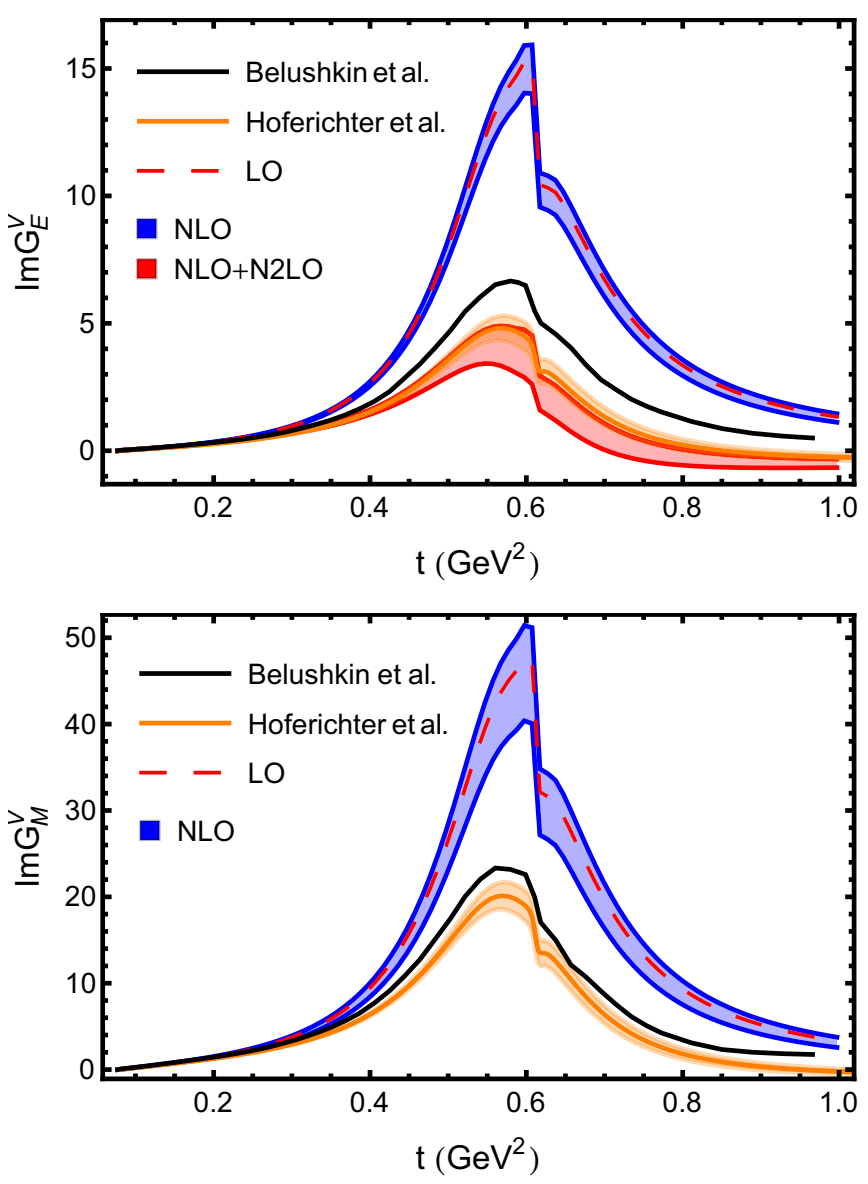

FIG. 5. DI $\chi$ EFT results for the spectral functions of the isovector nucleon EM FFs, Eqs. (7) and (8). Dashed line: LO approximation. Blue band: NLO approximation. Red band: NLO+pN2LO, estimated as described in in Sec. IIC. Orange band: Results of Roy-Steiner analysis [52]. Black line: Empirical dispersion analysis [55].

for perturbative $\chi$ EFT treatment and are included through the empirical pion FF in our approach. Note that the enhancement through $\left|F_{\pi}(t)\right|^{2}$ is large even near the two-pion threshold $t \sim 4 M_{\pi}^{2}$, cf. Sec. IID and Fig. 3. The convergence pattern of the spectral functions follows from that of the $\chi \mathrm{EFT}$ calculation of $J_{ \pm}^{1}(t)$. In both $\operatorname{Im} G_{E}$ and $\operatorname{Im} G_{M}$, the LO and NLO approximations are in good agreement with the Roy-Steiner results up to $t \sim 0.2 \mathrm{GeV}^{2}$. In $\operatorname{Im} G_{E}$ the negative pN2LO correction (estimated) is sufficient to reproduce the Roy-Steiner result up to $t \sim 1 \mathrm{GeV}^{2}$.

\section{B. Nucleon EM radii}

The nucleon's isovector electric and magnetic radii are given by the dispersion integrals

$$
\left\langle r^{2}\right\rangle_{i}^{V}=\frac{6}{\pi} \int_{4 M_{\pi}^{2}}^{\infty} d t^{\prime} \frac{\operatorname{Im} G_{i}^{V}\left(t^{\prime}\right)}{t^{\prime 2}} \quad(i=E, M) .
$$

The factor $1 / t^{\prime 2}$ ensures convergence of the integral over the range $t^{\prime} \lesssim 1 \mathrm{GeV}^{2}$; see Fig. 6 . The integrals can therefore be evaluated with the DI $\chi$ EFT spectral functions. Table I summarizes the DI $\chi$ EFT results for the isovector radii and compares 


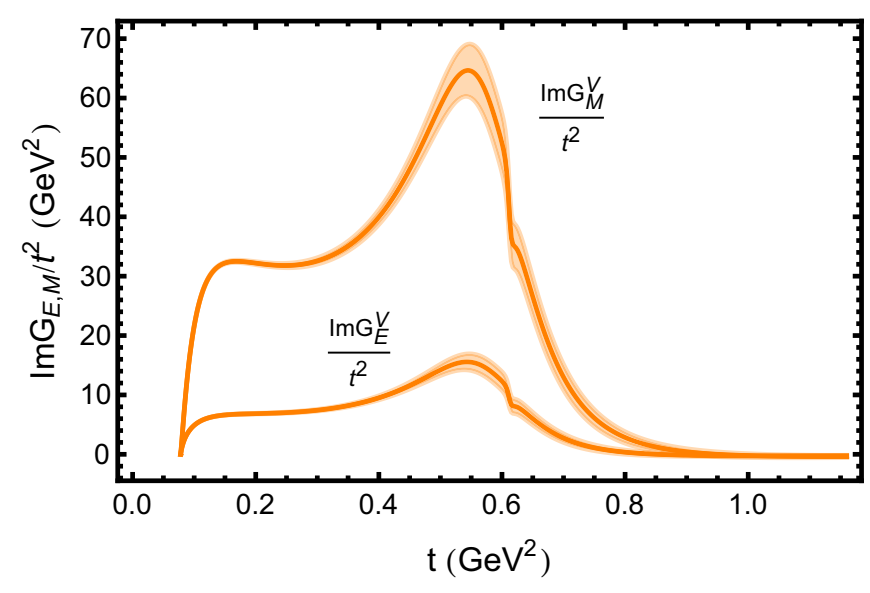

FIG. 6. The integrands of the dispersive integrals for the isovector nucleon electric and magnetic radii, Eq. (13), evaluated with the spectral functions of Ref. [52].

them with the results of other dispersive approaches and LQCD calculations. For the electric radius our NLO + pN2LO result agrees very well with the result of the Roy-Steiner analysis [52]. Our lower-order results overestimate this value, because the LO and NLO spectral functions are larger than the RoySteiner result around the $\rho$ peak. For the magnetic radius our result is larger by a factor $\sim 2$ than the phenomenological and LQCD results, because our magnetic spectral function is likewise too large around the $\rho$ peak.

DI $\chi$ EFT allows us to calculate the isovector nucleon EM FFs and radii, which are matrix elements of $G$-parity even operators. Experiments measure the individual proton and neutron FFs and radii. In view of the questions concerning the proton charge radius measurements, it is interesting to compare our results directly with the experimental results for the proton and neutron charge radii. To do so, we supplement the DI $\chi$ EFT results for the isovector spectral functions with an empirical parametrization of the isoscalar spectral functions. We use a two-pole parametrization with the $\omega$ pole at $M_{\omega}^{2}=0.61 \mathrm{GeV}^{2}$ and a second pole at $M_{2}^{2} \approx M_{\phi}^{2}=1 \mathrm{GeV}^{2}$ [15]:

$$
\operatorname{Im} G_{i}^{S}(t)=\pi\left[a_{i}^{\omega} \delta\left(t-M_{\omega}^{2}\right)+a_{i}^{(2)} \delta\left(t-M_{2}^{2}\right)\right] \quad(i=E, M),
$$

where the coefficients $a_{i}^{\omega}$ (including their uncertainties) are taken from the dispersive FF fit of Ref. [37], and the coefficients $a_{i}^{(2)}$ are adjusted to reproduce the total charge and magnetic moment. The second pole is an effective pole representing the overall strength of the spectral function at $t \sim 1 \mathrm{GeV}^{2}$; the details of the strength distribution at these values of $t$ are not important for estimating the nucleon radii. The proton and neutron radii obtained in this way are summarized in Table II. In the proton and neutron electric radii, the estimated uncertainty is dominated by the isoscalar component. (Note that we have a theoretical uncertainty estimate only for the isovector component calculated in DI $\chi \mathrm{EFT}$, and that the uncertainty in the isoscalar component is purely empirical.) Our results obtained with the NLO+pN2LO DI $\chi$ EFT calculation of the isovector radii are in agreement with the experimental values. In the magnetic radii the estimated uncertainty is likewise dominated by the isoscalar component. Note that the DI $\chi$ EFT calculation of the isovector magnetic spectral function does not include the N2LO corrections and strongly overestimates the empirical result (cf. Table I); this discrepancy is not reflected in the uncertainty estimate.

\section{Higher derivatives of EM form factors}

Higher derivatives of the nucleon EM FFs at $t=0$ are of interest for several reasons. In the experimental analysis, the values of higher derivatives allowed (or assumed) in fits of FF data at $t<0$ directly affect the extrapolation to $t=0$ and extraction of the nucleon charge radii; see Refs. [27-31] for details. In the theoretical studies reported here, higher derivatives of the FFs represent clean chiral observables that can be predicted almost model independently with minimal uncertainties. The comparison of low- and high-order derivatives reveals the presence of two dynamical scales in the nucleon FFs, which implies a surprisingly rich structure and should be incorporated into the experimental analysis.

In the context of the traditional representation of the FFs as Fourier transforms of three-dimensional spatial densities, the higher derivatives of the FFs at $t=0$ correspond to the higher $r^{2}$-weighted moments of the densities. The connection is given by [30]

$$
\begin{aligned}
G_{E}(t) & =1+\frac{\left\langle r^{2}\right\rangle_{E}}{3 !} t+\frac{\left\langle r^{4}\right\rangle_{E}}{5 !} t^{2}+\frac{\left\langle r^{6}\right\rangle_{E}}{7 !} t^{3}+\cdots, \\
\frac{G_{M}(t)}{\mu} & =1+\frac{\left\langle r^{2}\right\rangle_{M}}{3 !} t+\frac{\left\langle r^{4}\right\rangle_{M}}{5 !} t^{2}+\frac{\left\langle r^{6}\right\rangle_{M}}{7 !} t^{3}+\cdots,
\end{aligned}
$$

$$
\begin{aligned}
\frac{1}{n !} \frac{d^{n} G_{E}}{d t^{n}}(0) & =\frac{\left\langle r^{2 n}\right\rangle_{E}}{(2 n+1) !}, \\
\frac{1}{n ! \mu} \frac{d^{n} G_{M}}{d t^{n}}(0) & \left.=\frac{\left\langle r^{2 n}\right\rangle_{M}}{(2 n+1) !} \quad \text { (for either } p \text { or } n\right) .
\end{aligned}
$$

\begin{tabular}{|c|c|c|c|c|c|c|c|c|}
\hline & LO & NLO & $\mathrm{NLO}+\mathrm{pN} 2 \mathrm{LO}$ & $\begin{array}{c}\text { Lorenz } \\
\text { [26] }\end{array}$ & $\begin{array}{c}\text { Epstein } \\
\text { [53] }\end{array}$ & $\begin{array}{c}\text { Hoferichter } \\
\text { [52] }\end{array}$ & $\begin{array}{c}\text { LQCD } \\
{[54]}\end{array}$ & $\begin{array}{c}\text { Leupold } \\
\text { [33] }\end{array}$ \\
\hline$\left\langle r^{2}\right\rangle_{E}^{V}\left(\mathrm{fm}^{2}\right)$ & 0.98 & $(0.98,0.99)$ & $(0.33,0.43)$ & $0.416(8)$ & & $0.405(36)$ & $0.327(24)(15)$ & $(0.27,0.31)$ \\
\hline
\end{tabular}

Note that for the proton and neutron the magnetic radii are defined as the derivatives of the FFs divided by the magnetic

TABLE I. Isovector nucleon EM radii calculated in DI $\chi$ EFT (left columns) and in other approaches (right columns).

${ }^{\mathrm{a}}$ In the $\chi$ EFT calculation of Ref. [33] the $\operatorname{LEC~} c_{4}$ is adjusted to reproduce the magnetic radius. 
TABLE II. Left columns: Proton and neutron EM radii obtained from the DI $\chi$ EFT calculation of the isovector radii and the empirical parametrization of the isoscalar radii. Right columns: LQCD and experimental results. For the experimental values we quote the averages compiled by the Particle Data Group (upper and lower limits obtained by adding statistical and systematic errors) [56]. The smaller values correspond to the extraction from muonic hydrogen measurements; the larger values correspond to electronic hydrogen measurements; extractions from electron-proton scattering data using different methods have produced results supporting either value; see Ref. [56] for details.

\begin{tabular}{|c|c|c|c|c|c|}
\hline & LO & NLO & $\mathrm{NLO}+\mathrm{pN} 2 \mathrm{LO}$ & LQCD [54] & PDG [56] \\
\hline$\left\langle r^{2}\right\rangle_{E}^{n}\left(\mathrm{fm}^{2}\right)$ & $(-0.84,-0.47)$ & $(-0.88,-0.40)$ & $(-0.29,0.18)$ & $-0.038(34)(6)$ & $-0.1161(22)$ \\
\hline$\left\langle r^{2}\right\rangle_{M}^{n}\left(\mathrm{fm}^{2}\right)$ & $(1.29,1.64)$ & $(1.08,1.81)$ & & $0.586(58)(75)$ & $(0.73,0.76)$ \\
\hline
\end{tabular}

moments; this is not the case for the isovector and isoscalar components. In the following we quote results for the moments; they can be converted to FF derivatives through Eqs. (17) and (18). ${ }^{1}$

The higher moments of the isovector FFs are given by the dispersion integrals

$$
\frac{\left\langle r^{2 n}\right\rangle_{i}^{V}}{(2 n+1) !}=\frac{1}{\pi} \int_{4 M_{\pi}^{2}}^{\infty} d t^{\prime} \frac{\operatorname{Im} G_{i}^{V}\left(t^{\prime}\right)}{t^{\prime n+1}} \quad(i=E, M) .
$$

The factors $1 / t^{\prime n+1}$ strongly suppress contributions from large $t^{\prime}$ and render the integrals well convergent. The integrals can therefore be evaluated accurately using the DI $\chi$ EFT spectral functions. Figure 7 shows the integrands for the isovector electric FF derivatives with $n=\{1,2,3\}$; the curves are normalized to unit integral for each $n$ and show the relative distribution of strength in $t^{\prime}$. The distributions clearly indicate the presence of two dynamical scales: the $\rho$ meson mass $m_{\rho}^{2} \sim 0.6 \mathrm{GeV}^{2} \approx$ $30 M_{\pi}^{2}$ (the peak of the spectral function), and the two-pion threshold $4 M_{\pi}^{2}$ (the start of the spectral integral). The integrals receive contributions from both regions of $t^{\prime}$, and their relative importance changes with $n$. For a rough assessment we can take $M_{\rho}^{2} / 2=0.3 \mathrm{GeV}^{2}$ as the boundary between the two regions. For $n=1$ approximately $2 / 3$ of the integral comes from the region $t^{\prime}>M_{\rho}^{2} / 2$, and $1 / 3$ from $4 M_{\pi}^{2}<t<M_{\rho}^{2} / 2$. For $n=2$, each region contributes about $1 / 2$. For $n=3$ and higher, the near-threshold region dominates.

The presence of two dynamical scales in the isovector moments can also be demonstrated by considering the ratios of successive moments,

$$
\frac{\left\langle r^{2 n+2}\right\rangle_{i}^{V}}{(2 n+3) !} / \frac{\left\langle r^{2 n}\right\rangle_{i}^{V}}{(2 n+1) !} \quad(i=E, M)
$$

If the dispersion integral were dominated by a certain region of $t^{\prime}$, the value of the ratio Eq. (20) would be given by the

\footnotetext{
${ }^{1}$ The representation of FFs in terms of three-dimensional spatial densities is physically meaningful only for nonrelativistic systems. For relativistic systems such as the nucleon a proper spatial representation is provided by the two-dimensional transverse densities at fixed light-front time; see Ref. [57] for a review. We refer to the moments $\left\langle r^{2 n}\right\rangle$ only because this representation is used in the experimental literature, and use it only in the sense of a mathematical representation of the FF derivatives at $t=0$.
}

average of $1 / t^{\prime}$ over that region. The ratios thus directly reveal the effective values of $1 / t^{\prime}$ in the integral. Figure 8 shows the ratios of the isovector electric FF moments obtained with the DI $\chi$ EFT spectral functions. One sees that the ratios start with a value $\sim 1 / m_{\rho}^{2}$ at $n=1$ and increase to values $\sim 1 /\left(4 M_{\pi}^{2}\right)$ at large $n$.

The presence of two dynamical scales implies that the higher FF moments are of "unnatural" size, i.e., their values are very different from what one would estimate using the value of the lowest moment and a single-scale functional form of the FF. [In the dispersive representation such a single-scale form would be, e.g., a spectral function $\operatorname{Im} G_{i}^{V} \propto \delta\left(t-M_{\rho}^{2}\right)$, or derivatives thereof.] This conclusion relies only on general features of the dispersive representation and is insensitive to the details of the dynamical calculation presented here. It has has numerous consequences for the interpretation of the FF moments and the analysis of low- $t$ elastic scattering experiments, which will be elaborated below.

Table III shows the DI $\chi$ EFT results for the higher moments of the isovector FFs, $G_{E}^{V}$ and $G_{M}^{V}$. Because the dispersion integrals with $n \geqslant 2$ sample the spectral functions near threshold, the higher moments can be computed accurately and

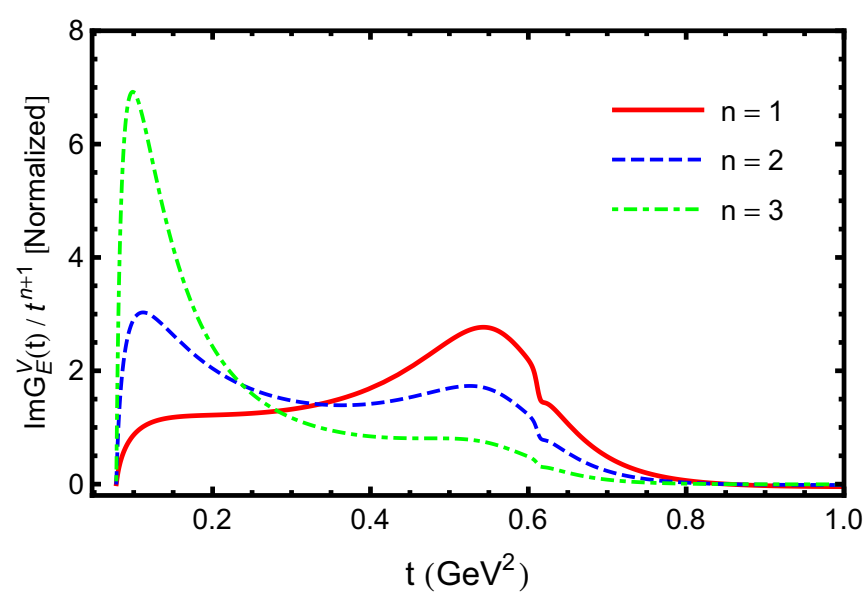

FIG. 7. Integrand of the dispersive integral for the moments of the isovector electric FF $G_{E}^{V}$, Eq. (19), for $n=1,2$, and 3, evaluated with the spectral functions of Ref. [52]. The plot shows the $t^{\prime}$ distributions divided by the value of the integral, i.e., normalized to unit area under the curves. 


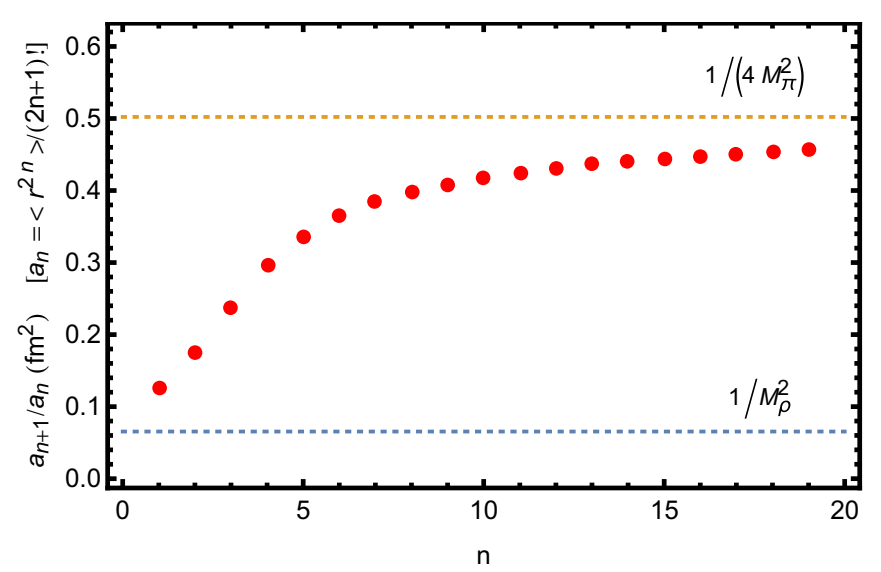

FIG. 8. Ratios of successive moments of the isovector electric FF $G_{E}^{V}$, Eq. (20), computed using the DI $\chi$ EFT isovector spectral functions (see Fig. 5). The horizontal lines indicate the values of the dynamical scales $1 / M_{\rho}^{2}$ and $1 /\left(4 M_{\pi}^{2}\right)$.

represent genuine predictions of our approach. This is seen in the intrinsic uncertainty estimates of Table III: with increasing $n$, the derivatives become less sensitive to higher-order chiral corrections. We emphasize that one should be careful in interpreting the numerical values of the individual moments in Table III, as they contain large factorial factors. The unnatural behavior of the higher moments should be demonstrated by taking ratios (see above) or comparing the moments to a reference FF (see below).

It is worth noting that the $\pi \pi$ rescattering effects included through the timelike pion FF play an important role even in the higher FF moments. The moments with $n \geqslant 3$ receive most of their contributions from the region $4 M_{\pi}^{2}<t^{\prime} \lesssim 10 M_{\pi}^{2}$ of the spectral integral Eq. (19) (see Fig. 7). The value of $\left|F_{\pi}\left(t^{\prime}\right)\right|^{2}$ is $\sim 1.3$ at $t^{\prime}=4 M_{\pi}^{2}$, and $\sim 2$ at $10 M_{\pi}^{2}$ (see Fig. 3 ). The enhancement compared to traditional direct $\chi$ EFT calculations of the spectral functions is therefore quite substantial (note that without the factor $\left|F_{\pi}\right|^{2}$ our results at LO and NLO would be identical to those of the direct calculation). Nevertheless our results for the higher moments agree with those of the direct $\chi$ EFT calculation of Ref. [35] within errors.

In the isoscalar FFs the strength of the spectral functions is located overwhelmingly at the $\omega$ meson mass. The higher

TABLE III. Higher moments of the isovector nucleon FFs calculated in DI $\chi$ EFT.

\begin{tabular}{lllr}
\hline \hline$G_{E}^{V}$ & & & \\
\hline & LO & NLO & NLO+pN2LO \\
\hline$\left\langle r^{4}\right\rangle\left(\mathrm{fm}^{4}\right)$ & 1.81 & $(1.72,1.86)$ & $(0.88,1.02)$ \\
$\left\langle r^{6}\right\rangle\left(\mathrm{fm}^{6}\right)$ & 9.86 & $(9.54,10.03)$ & $(6.68,7.16)$ \\
$\left\langle r^{8}\right\rangle\left(10^{2} \mathrm{fm}^{8}\right)$ & 1.40 & $(1.37,1.41)$ & $(1.17,1.20)$ \\
\hline$G_{M}^{V}$ & & & \\
\hline & & & \\
\hline$\left\langle r^{4}\right\rangle\left(\mathrm{fm}^{4}\right)$ & 6.49 & $(5.81,6.85)$ & \\
$\left\langle r^{6}\right\rangle\left(10 \mathrm{fm}^{6}\right)$ & 3.82 & $(3.53,3.98)$ & \\
$\left\langle r^{8}\right\rangle\left(10^{2} \mathrm{fm}^{8}\right)$ & 5.68 & $(5.38,5.84)$ & \\
\hline \hline
\end{tabular}

TABLE IV. Higher moments of the proton and neutron electric and magnetic FFs, calculated using the DI $\chi$ EFT results for the isovector moments and the empirical parametrization of the isoscalar FFs.

\begin{tabular}{|c|c|c|c|}
\hline \multicolumn{4}{|l|}{$G_{E}^{p}$} \\
\hline & LO & NLO & $\mathrm{NLO}+\mathrm{pN} 2 \mathrm{LO}$ \\
\hline$\left\langle r^{4}\right\rangle\left(\mathrm{fm}^{4}\right)$ & $(2.09,2.48)$ & $(2.00,2.53)$ & $(1.16,1.70)$ \\
\hline$\left\langle r^{6}\right\rangle\left(\mathrm{fm}^{6}\right)$ & $(10.8,11.7)$ & $(10.5,11.9)$ & $(7.59,9.00)$ \\
\hline$\left\langle r^{8}\right\rangle\left(10^{2} \mathrm{fm}^{8}\right)$ & $(1.44,1.48)$ & $(1.42,1.49)$ & $(1.21,1.29)$ \\
\hline \multicolumn{4}{|l|}{$G_{E}^{n}$} \\
\hline & $\mathrm{LO}$ & NLO & $\mathrm{NLO}+\mathrm{pN} 2 \mathrm{LO}$ \\
\hline$\overline{\left\langle r^{4}\right\rangle\left(\mathrm{fm}^{4}\right)}$ & $(-1.53,-1.13)$ & $(-1.58,-1.04)$ & $(-0.74,-0.20)$ \\
\hline$\left\langle r^{6}\right\rangle\left(\mathrm{fm}^{6}\right)$ & $(-8.94,-8.02)$ & $(-9.11,-7.71)$ & $(-6.24,-4.84)$ \\
\hline$\left\langle r^{8}\right\rangle\left(10^{2} \mathrm{fm}^{8}\right)$ & $(-1.35,-1.31)$ & $(-1.36,-1.29)$ & $(-1.15,-1.08)$ \\
\hline \multicolumn{4}{|l|}{$G_{M}^{p}$} \\
\hline & LO & NLO & \\
\hline$\overline{\left\langle r^{4}\right\rangle\left(\mathrm{fm}^{4}\right)}$ & $(2.38,2.68)$ & $(2.14,2.81)$ & \\
\hline$\left\langle r^{6}\right\rangle\left(10 \mathrm{fm}^{6}\right)$ & $(1.39,1.46)$ & $(1.29,1.52)$ & \\
\hline$\left\langle r^{8}\right\rangle\left(10^{2} \mathrm{fm}^{8}\right)$ & $(2.05,2.08)$ & $(1.94,2.13)$ & \\
\hline \multicolumn{4}{|l|}{$G_{M}^{n}$} \\
\hline & LO & NLO & \\
\hline$\overline{\left\langle r^{4}\right\rangle\left(\mathrm{fm}^{4}\right)}$ & $(3.30,2.87)$ & $(3.49,2.51)$ & \\
\hline$\left\langle r^{6}\right\rangle\left(10 \mathrm{fm}^{6}\right)$ & $(1.96,1.86)$ & $(2.04,1.71)$ & \\
\hline$\left\langle r^{8}\right\rangle\left(10^{2} \mathrm{fm}^{8}\right)$ & $(2.95,2.91)$ & $(3.04,2.75)$ & \\
\hline
\end{tabular}

moments are therefore governed by this single scale and are of natural size. This in turn implies that the higher moments of the proton and neutron FFs are dominated by the isovector component and can be inferred from our DI $\chi$ EFT results. Table IV shows our results for the moments of $G_{E}^{p}, G_{E}^{n}, G_{M}^{p}$, and $G_{M}^{n}$, obtained using the DI $\chi$ EFT results for the isovector moments and the empirical parametrization of the isoscalar FFs. We stress that the isoscalar information is used here only to demonstrate that the higher derivatives are dominated by the isovector component, and that the uncertainties associated with the isoscalar parametrization are irrelevant in the higher derivatives.

The theoretical results described here have implications for the analysis of electron-proton elastic scattering data at low $Q^{2} \equiv-t$ and the extraction of the proton charge radius. The overall behavior of $G_{E}^{p}$ in the region $0<Q^{2} \lesssim 1 \mathrm{GeV}^{2}$ is associated with a scale of the order of the vector meson mass $M_{V}^{2}(V=\rho, \omega)$. The first derivative of $G_{E}^{p}$ at $Q^{2}=0$ is of the order $1 / M_{V}^{2}$ and therefore appears natural, i.e., simple singlescale parametrizations of the finite- $Q^{2}$ data give a reasonable estimate of the first derivative. The higher derivatives, however, are governed by the scale $1 /\left(4 M_{\pi}^{2}\right)$ and appear unnatural. Single-scale parametrizations or "natural" powers of the first derivative give qualitatively wrong estimates of the higher derivatives. To illustrate the point we compare the order of magnitude of the higher derivatives obtained from DI $\chi$ EFT with the ones of the dipole parametrization

$$
G_{E}^{p}(t)[\text { dipole }]=\Lambda^{4} /\left(t-\Lambda^{2}\right)^{2},
$$

which provides a good overall description of the FF data at $0<Q^{2} \lesssim 1 \mathrm{GeV}^{2}$ with $\Lambda^{2} \approx 0.71 \mathrm{GeV}^{2}$. Figure 9 shows the 


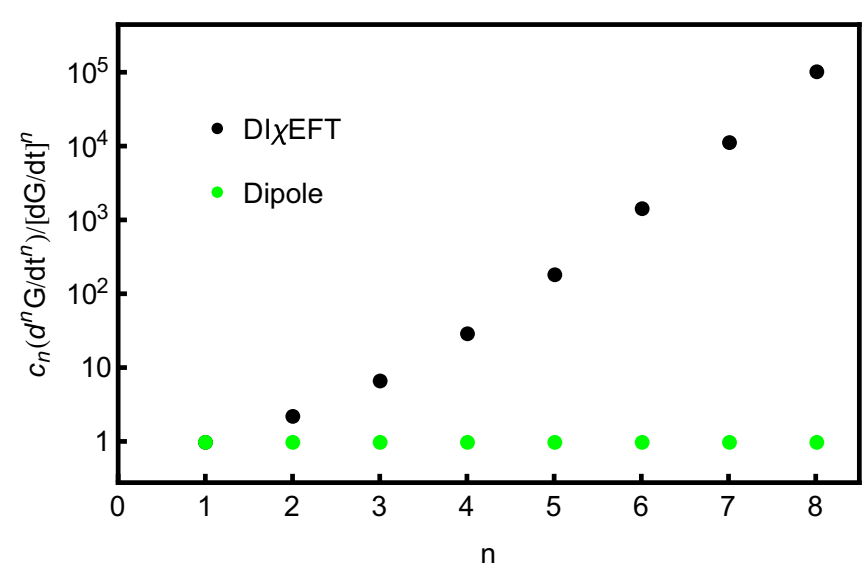

FIG. 9. The normalized ratios of the $n$th derivative of the proton electric FF $G_{E}^{p}$ and the $n$th power of the first derivative, Eq. (22). The ratios are normalized such that their values are unity for the dipole FF Eq. (21).

ratios

$$
c_{n} \frac{d^{n} G_{E}^{p}}{d t^{n}}(0) /\left[\frac{d^{n} G_{E}^{p}}{d t^{n}}(0)\right]^{n}, \quad c_{n} \equiv \frac{2^{n}}{(n+1) !},
$$

as obtained with the DI $\chi$ EFT results. The coefficients $c_{n}$ are defined such that for the dipole FF Eq. (21) the ratio is equal to unity for all $n$. The ratio Eq. (22) therefore indicates how strongly the actual higher derivatives deviate from the singlescale estimate based on the dipole form. One sees that the ratio is $\sim 10^{2}$ for $n=4$, and reaches values $\sim 10^{5}$ for $n=8$. It shows the striking consequences of the two dynamical scales in the higher FF derivatives, as implied by their dispersive representation. A similar observation regarding the ratio of the actual FF moments to the dipole was made in the context of an empirical analysis in Ref. [59].

The values of the higher derivatives of $G_{E}^{p}$ and their impact on the $Q^{2} \rightarrow 0$ extrapolation are presently the subject of intense discussions [27-31]. Fits to the low- $Q^{2}$ FF data with different classes of functions (polynomials, rational functions) give widely different values of the second and higher derivatives; see Table $\mathrm{V}$ for a compilation of recent results. The DI $\chi$ EFT results are broadly consistent with the range of empirical values. An analysis of FF data incorporating theoretical constraints from DI $\chi$ EFT will be the subject of a future study [60]. For reference we quote in Appendix B the numerical values of the DI $\chi$ EFT moments of $G_{E}^{p}$ up to $n=20$. While the individual values have little physical significance, their order of magnitude and collective behavior could be compared with the pattern and observed in higher-order polynomial fits.

The unnatural behavior of the higher FF derivatives is a consequence of analyticity and the singularities of the $\pi N$ Born amplitudes, which govern the dependence of the spectral function in the near-threshold region. Any dispersive description that includes the correct near-threshold dependence and the enhancement of the spectral function in the $\rho$ meson region should reproduce this behavior. Dispersive fits to the low- $Q^{2}$ FF data should therefore be able to provide reliable results for the higher FF derivatives [26,37].

\section{Spacelike EM form factors}

The DI $\chi$ EFT approach also allows us to calculate the nucleon FFs at finite $t<0$, where they are measured in $e N$ elastic scattering experiments. For the isovector FFs we use the twice-subtracted dispersion relations

$$
\begin{aligned}
G_{i}^{V}(t)= & G_{i}^{V}(0)+t \frac{d G_{i}^{V}}{d t}(0) \\
& +\frac{t^{2}}{\pi} \int_{4 M_{\pi}^{2}}^{\infty} d t^{\prime} \frac{\operatorname{Im} G_{i}^{V}\left(t^{\prime}\right)}{t^{\prime 2}\left(t^{\prime}-t\right)} \quad(i=E, M)
\end{aligned}
$$

Here the FFs at $t=0$ (charge and magnetic moment) and the first derivatives (electric and magnetic radii) are taken as input, and the dispersion relations predict the $t$ dependence starting from the second order. The integrals in Eq. (23) are well convergent and can be evaluated with the DI $\chi$ EFT spectral functions. For the isoscalar FF we use the empirical parametrization Eq. (14), which imposes the correct value of the FF at $t=0$. Combining the two we predict the individual proton and neutron FFs. Figure 10 summarizes the results. The estimated uncertainties are dominated by those of the isoscalar component, for which we have only the empirical parametrization. One observes the following:

(a) In the electric FFs $G_{E}^{p}$ and $G_{E}^{n}$, the LO and NLO approximations describe the experimental data only up to $Q^{2} \sim 0.1 \mathrm{GeV}^{2}$, while the NLO+pN2LO approximations show good agreement with the data up to $Q^{2} \sim 0.5 \mathrm{GeV}^{2}$. This reflects the improvement of the isovector electric spectral function due to the pN2LO corrections; see Figs. 4 and 5.

(b) In the magnetic FFs $G_{M}^{p}$ and $G_{M}^{n}$, our results describe the data up to $Q^{2} \sim 0.2 \mathrm{GeV}^{2}$. In this channel the N2LO corrections cannot be estimated using the method of Sec. II C.

TABLE V. Higher moments of $G_{E}^{p}$ extracted from recent fits to low- $Q^{2}$ FF data using different classes of functions.

\begin{tabular}{lcccrr}
\hline \hline$G_{E}^{p}$ & & & & \\
\hline & $\begin{array}{c}\text { Standard } \\
\text { Dipole }\end{array}$ & $\begin{array}{c}\text { Bernauer } \\
{[58]}\end{array}$ & $\begin{array}{c}\text { Horbatsch } \\
{[30]}\end{array}$ & $\begin{array}{c}\text { Higinbotham } \\
\text { Rational [28] }\end{array}$ & $\begin{array}{c}\text { Higinbotham } \\
\text { Power series [28] }\end{array}$ \\
\hline$\left\langle r^{4}\right\rangle\left(\mathrm{fm}^{4}\right)$ & 1.08 & 2.63 & 0.60 & 1.38 & $\begin{array}{c}\text { Sick } \\
{[31]}\end{array}$ \\
$\left\langle r^{6}\right\rangle\left(\mathrm{fm}^{6}\right)$ & 3.30 & 26.93 & 5.00 & 5.68 & 2.01 \\
$\left\langle r^{8}\right\rangle\left(\mathrm{fm}^{8}\right)$ & 16.2 & 408.12 & 99.36 & 40.06 & 24.68 \\
\hline \hline
\end{tabular}



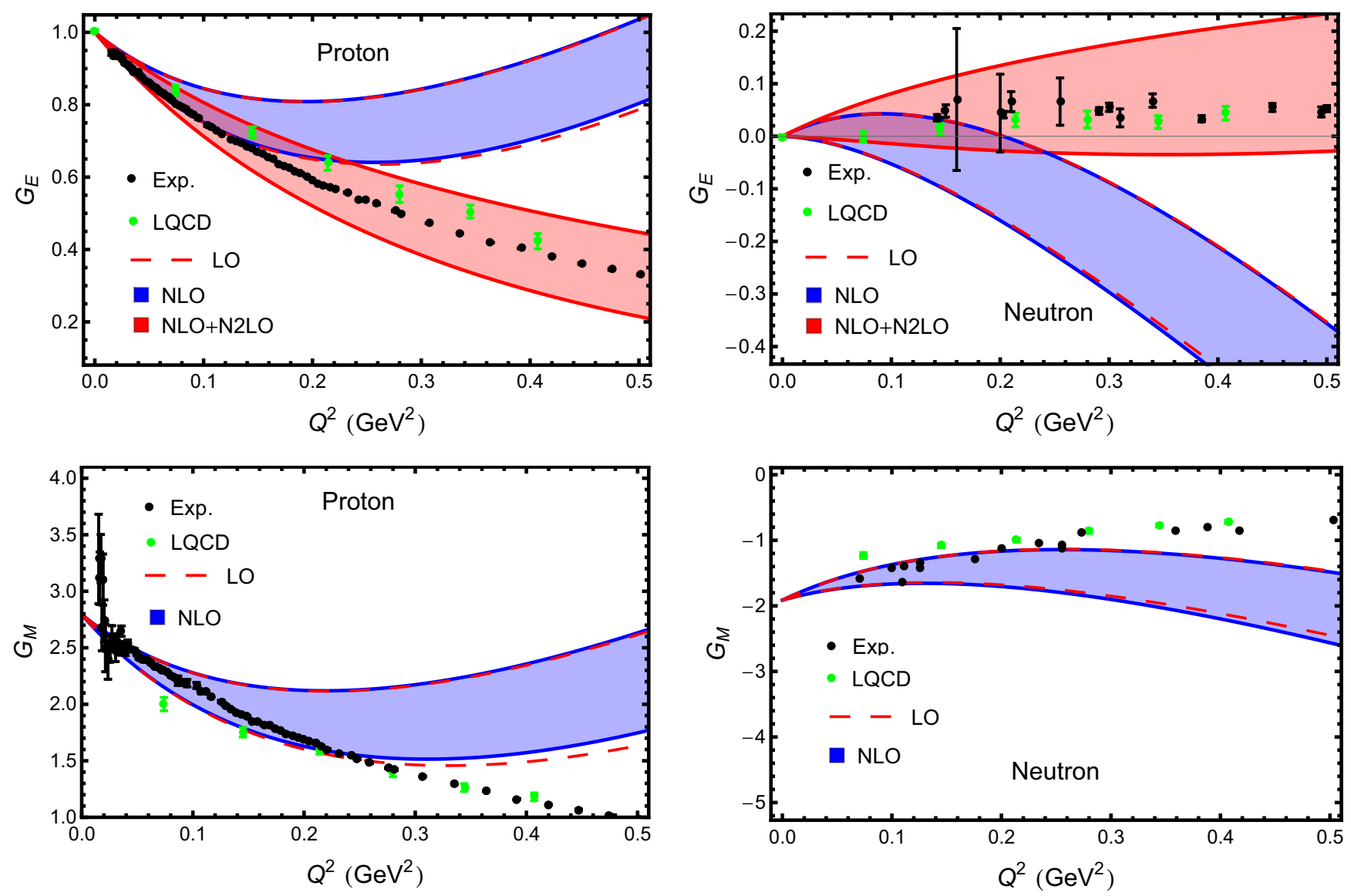

FIG. 10. DI $\chi$ EFT predictions for the proton and neutron electric and magnetic FFs, obtained with the twice-subtracted dispersion relation for the isovector FF, Eq. (23), and the empirical parametrization of the isoscalar FF Eq. (14). The results are compared to the experimental data of the A1 Collaboration [1,2] and the LQCD results from the ETM Collaboration [54].

Altogether we obtain a very satisfactory description of the nucleon EM FFs with our dynamical approach.

\section{SUMMARY}

This work reports a study of the nucleon EM FFs at momentum transfers $|t| \ll 1 \mathrm{GeV}^{2}$ using a new method combining $\chi$ EFT and dispersion analysis (DI $\chi$ EFT). The isovector spectral functions on the two-pion cut are constructed through the elastic unitarity condition. The $N / D$ method is used to separate effects of $\pi \pi$ rescattering from the coupling of the $\pi \pi$ system to the nucleon. $\chi$ EFT is employed to calculate the real functions $J_{ \pm}^{1}(t)$ describing the $\pi \pi$ coupling to the nucleon, which are free of $\pi \pi$ rescattering effects, resulting in good convergence. $\pi \pi$ rescattering effects are included through the timelike pion $\mathrm{FF}\left|F_{\pi}(t)\right|^{2}$, which can be taken from $e^{+} e^{-}$ annihilation data or LQCD calculations. The new organization is consistent with basic principles of $\chi \mathrm{EFT}$ and represents a major improvement over traditional direct calculations of the spectral functions. It allows us to calculate the isovector spectral functions up to $t \sim 1 \mathrm{GeV}^{2}$ (including the $\rho$ meson region) with controlled accuracy. With these spectral functions we are able to evaluate elements of the FFs (radii, higher derivatives, $t$ dependence in the spacelike region) using wellconvergent subtracted dispersion relations.
The new method permits a realistic description of the low- $t$ nucleon FFs and their derivatives. While the basic features of the FFs are rooted in analyticity and have been studied earlier in empirical dispersion theory, the new aspect is that the spectral functions can now be computed in a $\chi$ EFT framework with controlled accuracy. It makes it possible to represent the information content of the nucleon FFs in the form of a few physical masses and LECs, resulting in a significant reduction of complexity. It also enables new interpretations of FFs in terms of spatial densities $[14,15]$ and a space-time picture of the chiral processes in peripheral nucleon structure [61-63].

Our study shows that the derivatives of the EM FFs involve two dynamical scales. The first derivative is governed by the scale $1 / M_{V}^{2}$, while higher derivatives are governed by the scale $1 /\left(4 M_{\pi}^{2}\right) \gg 1 / M_{V}^{2}$ and therefore appear unnaturally large. The rich structure attests to the fact that, through analyticity and the dispersion relations, the FFs of the nucleon are connected to its hadronic couplings and excitation spectrum and reflect the multiple dynamical scales characterizing the latter. The DI $\chi$ EFT calculations provide an explicit realization of this general feature. While the predicted pattern of the higher moments is likely far beyond what can be extracted from experimental data, our analytic functions can be used in Monte Carlo simulations to study how well the first few moments can be extracted under realistic conditions. Our numerical 
estimates of the higher derivatives can be used in an empirical analysis based on bounded least-squares regression. An analysis incorporating theoretical constraints from DI $\chi$ EFT is in progress [60].

The DI $\chi$ EFT FF calculations described here could be extended in several directions. The method could be applied to the $N-\Delta$ transition FFs as well as the EM FFs of the $\Delta$ itself, which are defined rigorously in the context of $S$-matrix theory (as poles in the $N \rightarrow \pi N$ and $\pi N \rightarrow \pi N$ EM transition amplitudes) and have been studied in relativistic $\chi$ EFT $[64,65]$. The method could also be applied to nucleon FFs of other $G$-parity-even operators, such as the energy-momentum tensor or higher moments of the GPDs. Finally, one might contemplate extending the DI $\chi$ EFT approach to nucleon FFs of $G$-parity odd operators with a three-pion cut, using methods of three-body elastic unitarity that are presently being developed for the analysis of meson decays and LQCD calculations $[66,67]$.

The present study demonstrates the potential of $\chi$ EFT to yield fully predictive results for conventional nucleon structure observables. The same approach can be applied to hadronic structure elements appearing in searches for physics beyond the standard model; see, e.g., Refs. [68-70].

\section{ACKNOWLEDGMENTS}

We are indebted to C. Alexandrou, J. Bernauer, and A. Pineda for providing us with experimental and theoretical data shown in the tables figures, and to D. Higinbotham for valuable discussions and help with the compilation of data and references. This material is based upon work supported by the US Department of Energy, Office of Science, Office of Nuclear Physics under Contract No. DE-AC05-06OR23177. This work was also supported by the Spanish Ministerio de Economía y Competitividad and European FEDER funds under Contract No. FPA2016-77313-P.

\section{APPENDIX A: $\boldsymbol{J}$ FUNCTIONS IN $\chi$ EFT}

In this Appendix we list the $\chi \mathrm{EFT}$ expressions for the real functions $J_{ \pm}^{1}(t)$, Eq. (9), which appear in the $N / D$ representation of the elastic unitarity condition and are used in the analytical and numerical studies described in the text. In the following $4 M_{\pi}^{2}<t<4 m_{N}^{2}$, and

$$
k_{\mathrm{cm}}=\sqrt{t / 4-M_{\pi}^{2}}, \quad \tilde{p}_{\mathrm{cm}}=\sqrt{m_{N}^{2}-t / 4}
$$

are, respectively, the physical pion $\mathrm{CM}$ momentum and the unphysical nucleon CM momentum in the $\pi \pi \rightarrow N \bar{N}$ process. The functions resulting from the Weinberg-Tomozawa contact term [Fig. 1(b)] and the $N$ Born term [Fig. 1(a)] are

$$
\begin{aligned}
& J_{+}^{1}(t)[\text { LO }, \text { cont }]=\frac{m_{N}}{24 \pi f_{\pi}^{2}}, \\
& J_{-}^{1}(t)[\text { LO }, \text { cont }]=\frac{\sqrt{2}}{24 \pi f_{\pi}^{2}},
\end{aligned}
$$

$$
\begin{aligned}
J_{+}^{1}(t)[\mathrm{LO}, N]= & \frac{g_{A}^{2} m_{N}^{3} A_{N}^{2}}{16 \pi f_{\pi}^{2} \widetilde{p}_{\mathrm{cm}}^{3} k_{\mathrm{cm}}^{3}}\left(-\arctan x_{N}+x_{N}\right) \\
& -\frac{g_{A}^{2} m_{N}}{24 \pi f_{\pi}^{2}}, \\
J_{-}^{1}(t)[\mathrm{LO}, N]= & \frac{\sqrt{2} g_{A}^{2} m_{N}^{2} A_{N}^{2}}{32 \pi f_{\pi}^{2} \widetilde{p}_{\mathrm{cm}}^{3} k_{\mathrm{cm}}^{3}}\left[\left(x_{N}^{2}+1\right) \arctan x_{N}-x_{N}\right] \\
& -\frac{\sqrt{2} g_{A}^{2}}{24 \pi f_{\pi}^{2}}, \\
A_{N} \equiv & t / 2-M_{\pi}^{2}, \\
x_{N} \equiv & \frac{2 k_{\mathrm{cm}} \widetilde{p}_{\mathrm{cm}}}{A_{N}} \\
= & \frac{2 \sqrt{t / 4-M_{\pi}^{2}} \sqrt{m_{N}^{2}-t / 4}}{t / 2-M_{\pi}^{2}} .
\end{aligned}
$$

The contributions of the $\Delta$ Born term [Fig. 1(c)] are

$$
\begin{aligned}
J_{+}^{1}(t)[\mathrm{LO}, \Delta]= & \frac{h_{A}^{2} A_{\Delta}\left(2 \widetilde{p}_{\mathrm{cm}}^{2} F-A_{\Delta} m_{N} G\right)}{192 \pi f_{\pi}^{2} \widetilde{p}_{\mathrm{cm}}^{3} k_{\mathrm{cm}}^{3}} \\
& \times\left(\arctan x_{\Delta}-x_{\Delta}\right)+\frac{h_{A}^{2} D_{\Delta+}}{432 \pi f_{\pi}^{2} m_{\Delta}^{2}}, \\
J_{-}^{1}(t)[\mathrm{LO}, \Delta]= & \frac{\sqrt{2} h_{A}^{2} A_{\Delta}^{2} G}{384 \pi f_{\pi}^{2} \widetilde{p}_{\mathrm{cm}}^{3} k_{\mathrm{cm}}^{3}}\left[\left(x_{\Delta}^{2}+1\right) \arctan x_{\Delta}-x_{\Delta}\right] \\
& +\frac{\sqrt{2} h_{A}^{2} D_{\Delta-}}{864 \pi f_{\pi}^{2} m_{\Delta}^{2}}, \\
A_{\Delta} \equiv & t / 2-M_{\pi}^{2}+m_{\Delta}^{2}-m_{N}^{2}, \\
x_{\Delta} \equiv & \frac{2 k_{\mathrm{cm}} \tilde{p}_{\mathrm{cm}}}{A_{\Delta}} \\
= & \frac{2 \sqrt{t / 4-M_{\pi}^{2}} \sqrt{m_{N}^{2}-t / 4}}{t / 2-M_{\pi}^{2}+m_{\Delta}^{2}-m_{N}^{2}} .
\end{aligned}
$$

The functions $F$ and $G$ appearing in the first terms of Eqs. (A8) and (A9) are [16]

$$
\begin{aligned}
F & \equiv \alpha\left(m_{\Delta}+m_{N}\right)+\frac{\beta}{3}\left(m_{\Delta}-m_{N}\right), \\
G & \equiv-\alpha+\frac{\beta}{3}, \\
\alpha & \equiv \frac{t}{2}-m_{N}^{2}+\frac{\left(m_{\Delta}^{2}+m_{N}^{2}-M_{\pi}^{2}\right)^{2}}{4 m_{\Delta}^{2}}, \\
\beta & \equiv\left(m_{N}+\frac{m_{\Delta}^{2}+m_{N}^{2}-M_{\pi}^{2}}{2 m_{\Delta}}\right)^{2} ;
\end{aligned}
$$

they are the invariant amplitudes of $\pi N$ scattering at $t>4 M_{\pi}^{2}$ and $s=m_{\Delta}^{2}$ in the conventions of Ref. [63]. The functions $D_{\Delta+}$ and $D_{\Delta-}$ appearing in the second terms in Eqs. (A8) and (A9) are

$$
\begin{aligned}
& D_{\Delta+}=m_{N}^{3}+2 m_{N}^{2} m_{\Delta}+m_{N} m_{\Delta}^{2}-m_{N} M_{\pi}^{2}+\left(m_{N}-m_{\Delta}\right) t, \\
& D_{\Delta-}=-10 m_{N}^{2}-4 m_{N} m_{\Delta}+2 m_{\Delta}^{2}-2 M_{\pi}^{2}+5 t .
\end{aligned}
$$


The $J$ functions resulting from the $N$ and $\Delta$ Born terms have logarithmic left-hand cuts starting at

$$
\left.\begin{array}{ll}
N: & t<4 M_{\pi}^{2}-M_{\pi}^{4} / m_{N}^{2} \\
\Delta: & t<4 M_{\pi}^{2}-\left(m_{\Delta}^{2}-m_{N}^{2}+M_{\pi}^{2}\right)^{2} / m_{\Delta}^{2}
\end{array}\right\} .
$$

The singularity results from the intermediate baryon lines going on mass shell and corresponds to the left-hand cut of the $\pi \pi \rightarrow N \bar{N}$ PWA. The singularity is contained in the inverse tangent functions in Eqs. (A4), (A5), (A8), and (A5), which have logarithmic branch points at $x_{N, \Delta}= \pm i$. The $J$ functions do not have a right-hand cut at $t>4 M_{\pi}^{2}$, in accordance with their definition within the $N / D$ method, Eqs. (9) and (10). While the expressions in Eqs. (A4), (A5), (A8), and (A9) contain prefactors with inverse powers of $k_{\mathrm{cm}}$, they are in fact regular in the limit $k_{\mathrm{cm}} \rightarrow 0$, because the expressions in parentheses/brackets depending on $x_{N}$ or $x_{\Delta}$ vanish in the limit: $x_{N, \Delta}=\mathcal{O}\left(k_{\mathrm{cm}}\right)$. Further properties of the $J$ functions are discussed in Ref. [16].

The masses and coupling constants used in evaluating the LO expressions are the standard values for the SU(2) flavor group [16]: $M_{\pi}=139 \mathrm{MeV}, f_{\pi}=93 \mathrm{MeV}, m_{N}=$ $939 \mathrm{MeV}, g_{A}=1.27$, and $m_{\Delta}=1232 \mathrm{MeV}, h_{A}=2.85$.

The contributions of the NLO contact term in the $\pi N$ amplitude are

$$
\begin{aligned}
& J_{+}^{1}(t)[\mathrm{NLO}, \mathrm{cont}]=\frac{c_{4} t}{24 \pi f_{\pi}^{2}}, \\
& J_{-}^{1}(t)[\mathrm{NLO}, \mathrm{cont}]=\frac{\sqrt{2} m_{N} c_{4}}{6 \pi f_{\pi}^{2}} .
\end{aligned}
$$

The value of the LEC $c_{4}$, determined by the procedure described in Sec. IIC, is given in Eq. (11).

\section{APPENDIX B: HIGHER DERIVATIVES OF PROTON ELECTRIC FORM FACTOR}

For reference we present in Table VI our numerical estimates of the higher moments of the proton electric FF,
TABLE VI. Higher-order moments of $G_{E}^{p}$, obtained by combining the DI $\chi$ EFT calculation of the isovector derivatives with an empirical estimate of the isoscalar derivatives.

\begin{tabular}{lccc}
\hline \hline$G_{E}^{p}$ & & & \\
\hline & LO & NLO & NLO+pN2LO \\
\hline$\left\langle r^{4}\right\rangle\left(\mathrm{fm}^{4}\right)$ & $(2.09,2.48)$ & $(2.00,2.53)$ & $(1.16,1.70)$ \\
$\left\langle r^{6}\right\rangle\left(\mathrm{fm}^{6}\right)$ & $(10.8,11.7)$ & $(10.5,11.8)$ & $(7.59,9.00)$ \\
$\left\langle r^{8}\right\rangle\left(10^{2} \mathrm{fm}^{8}\right)$ & $(1.44,1.48)$ & $(1.42,1.49)$ & $(1.21,1.29)$ \\
$\left\langle r^{10}\right\rangle\left(10^{3} \mathrm{fm}^{10}\right)$ & $(4.21,4.24)$ & $(4.18,4.26)$ & $(3.86,3.94)$ \\
$\left\langle r^{12}\right\rangle\left(10^{5} \mathrm{fm}^{12}\right)$ & $(2.13,2.13)$ & $(2.12,2.14)$ & $(2.02,2.04)$ \\
$\left\langle r^{14}\right\rangle\left(10^{7} \mathrm{fm}^{14}\right)$ & $(1.60,1.61)$ & $(1.60,1.61)$ & $(1.55,1.56)$ \\
$\left\langle r^{16}\right\rangle\left(10^{9} \mathrm{fm}^{16}\right)$ & $(1.66,1.66)$ & $(1.66,1.67)$ & $(1.61,1.62)$ \\
$\left\langle r^{18}\right\rangle\left(10^{11} \mathrm{fm}^{18}\right)$ & $(2.25,2.25)$ & $(2.25,2.26)$ & $(2.20,2.21)$ \\
$\left\langle r^{20}\right\rangle\left(10^{13} \mathrm{fm}^{20}\right)$ & $(3.86,3.86)$ & $(3.85,3.87)$ & $(3.79,3.80)$ \\
$\left\langle r^{22}\right\rangle\left(10^{15} \mathrm{fm}^{22}\right)$ & $(8.15,8.15)$ & $(8.13,8.16)$ & $(8.00,8.03)$ \\
$\left\langle r^{24}\right\rangle\left(10^{18} \mathrm{fm}^{24}\right)$ & $(2.08,2.08)$ & $(2.07,2.08)$ & $(2.04,2.05)$ \\
$\left\langle r^{26}\right\rangle\left(10^{20} \mathrm{fm}^{26}\right)$ & $(6.28,6.28)$ & $(6.27,6.28)$ & $(6.18,6.20)$ \\
$\left\langle r^{28}\right\rangle\left(10^{23} \mathrm{fm}^{28}\right)$ & $(2.22,2.22)$ & $(2.22,2.22)$ & $(2.19,2.20)$ \\
$\left\langle r^{30}\right\rangle\left(10^{25} \mathrm{fm}^{30}\right)$ & $(9.11,9.11)$ & $(9.09,9.11)$ & $(8.99,9.01)$ \\
$\left\langle r^{32}\right\rangle\left(10^{28} \mathrm{fm}^{32}\right)$ & $(4.27,4.27)$ & $(4.27,4.28)$ & $(4.22,4.23)$ \\
$\left\langle r^{34}\right\rangle\left(10^{31} \mathrm{fm}^{34}\right)$ & $(2.28,2.28)$ & $(2.27,2.28)$ & $(2.25,2.26)$ \\
$\left\langle r^{36}\right\rangle\left(10^{34} \mathrm{fm}^{36}\right)$ & $(1.37,1.37)$ & $(1.37,1.37)$ & $(1.35,1.36)$ \\
$\left\langle r^{38}\right\rangle\left(10^{36} \mathrm{fm}^{38}\right)$ & $(9.20,9.20)$ & $(9.19,9.21)$ & $(9.10,9.12)$ \\
$\left\langle r^{40}\right\rangle\left(10^{39} \mathrm{fm}^{40}\right)$ & $(6.88,6.88)$ & $(6.87,6.89)$ & $(6.81,6.83)$ \\
\hline \hline
\end{tabular}

obtained by combining the DI $\chi$ EFT calculation of the isovector moments with the empirical estimate of the isoscalar moments based on Eq. (14). While individual higher moments have little physical significance and cannot realistically be extracted from the data, the order of magnitude and collective behavior of our results could be compared with the patterns observed in fits to FF data $[1,2,26-31,58]$.
[1] J. C. Bernauer et al. (A1 Collaboration), Phys. Rev. Lett. 105, 242001 (2010).

[2] J. C. Bernauer et al. (A1 Collaboration), Phys. Rev. C 90, 015206 (2014).

[3] M. Mihovilovič et al., Phys. Lett. B 771, 194 (2017).

[4] C. B. Crawford et al., Phys. Rev. Lett. 98, 052301 (2007).

[5] M. Paolone et al., Phys. Rev. Lett. 105, 072001 (2010).

[6] X. Zhan et al., Phys. Lett. B 705, 59 (2011).

[7] C. F. Perdrisat, V. Punjabi, and M. Vanderhaeghen, Prog. Part. Nucl. Phys. 59, 694 (2007).

[8] V. Punjabi, C. F. Perdrisat, M. K. Jones, E. J. Brash, and C. E. Carlson, Eur. Phys. J. A 51, 79 (2015).

[9] Z. Ye, J. Arrington, R. J. Hill, and G. Lee, Phys. Lett. B 777, 8 (2018).

[10] R. Pohl et al., Nature (London) 466, 213 (2010).

[11] C. E. Carlson, Prog. Part. Nucl. Phys. 82, 59 (2015).

[12] J. J. Krauth et al., arXiv:1706.00696.

[13] A. H. Gasparian (PRad Collaboration), JPS Conf. Proc. 13, 020052 (2017).
[14] C. Granados and C. Weiss, J. High Energy Phys. 01 (2014) 092.

[15] J. M. Alarcón, A. N. Hiller Blin, M. J. Vicente Vacas, and C. Weiss, Nucl. Phys. A 964, 18 (2017).

[16] J. M. Alarcón and C. Weiss, Phys. Rev. C 96, 055206 (2017).

[17] W. R. Frazer and J. R. Fulco, Phys. Rev. 117, 1603 (1960).

[18] W. R. Frazer and J. R. Fulco, Phys. Rev. 117, 1609 (1960).

[19] J. Gasser, M. E. Sainio, and A. Svarc, Nucl. Phys. B 307, 779 (1988).

[20] V. Bernard, N. Kaiser, and Ulf-G. Meißner, Nucl. Phys. A 611, 429 (1996).

[21] T. Becher and H. Leutwyler, Eur. Phys. J. C 9, 643 (1999).

[22] B. Kubis and Ulf-G. Meißner, Nucl. Phys. A 679, 698 (2001).

[23] N. Kaiser, Phys. Rev. C 68, 025202 (2003).

[24] G. Höhler, in Pion Nucleon Scattering. Part 2: Methods and Results of Phenomenological Analyses, Landolt-Börnstein 9b2, edited by H. Schopper (Springer, Berlin, 1983).

[25] M. Hoferichter, C. Ditsche, B. Kubis, and Ulf-G. Meißner, J. High Energy Phys. 06 (2012) 063. 
[26] I. T. Lorenz, H.-W. Hammer, and U.-G. Meißner, Eur. Phys. J. A 48, 151 (2012).

[27] G. Lee, J. R. Arrington, and R. J. Hill, Phys. Rev. D 92, 013013 (2015).

[28] D. W. Higinbotham, Al A. Kabir, V. Lin, D. Meekins, B. Norum, and B. Sawatzky, Phys. Rev. C 93, 055207 (2016).

[29] K. Griffioen, C. Carlson, and S. Maddox, Phys. Rev. C 93, 065207 (2016).

[30] M. Horbatsch, E. A. Hessels, and A. Pineda, Phys. Rev. C 95, 035203 (2017).

[31] I. Sick and D. Trautmann, Phys. Rev. C 95, 012501 (2017).

[32] C. Granados, S. Leupold, and E. Perotti, Eur. Phys. J. A 53, 117 (2017).

[33] S. Leupold, Eur. Phys. J. A 54, 1 (2018).

[34] T. Bauer, J. C. Bernauer, and S. Scherer, Phys. Rev. C 86, 065206 (2012).

[35] C. Peset and A. Pineda, Nucl. Phys. B 887, 69 (2014).

[36] G. Hohler et al., Nucl. Phys. B 114, 505 (1976).

[37] M. A. Belushkin, H.-W. Hammer, and Ulf-G. Meißner, Phys. Rev. C 75, 035202 (2007).

[38] K. M. Watson, Phys. Rev. 95, 228 (1954).

[39] G. Hohler and E. Pietarinen, Phys. Lett. B 53, 471 (1975).

[40] G. F. Chew and S. Mandelstam, Phys. Rev. 119, 467 (1960).

[41] H. B. Meyer, Phys. Rev. Lett. 107, 072002 (2011).

[42] J. M. Alarcón, J. M. Camalich, and J. A. Oller, Ann. Phys. (NY) 336, 413 (2013).

[43] V. Pascalutsa, Phys. Rev. D 58, 096002 (1998).

[44] V. Pascalutsa and R. Timmermans, Phys. Rev. C 60, 042201 (1999).

[45] V. Pascalutsa, Phys. Lett. B 503, 85 (2001).

[46] H. Krebs, E. Epelbaum, and Ulf-G. Meißner, Phys. Rev. C 80, 028201 (2009).

[47] T. Fuchs, J. Gegelia, G. Japaridze, and S. Scherer, Phys. Rev. D 68, 056005 (2003).

[48] V. Bernard, N. Kaiser, and Ulf G. Meißner, Nucl. Phys. A 615, 483 (1997).
[49] D. Siemens et al., Phys. Lett. B 770, 27 (2017).

[50] G. J. Gounaris and J. J. Sakurai, Phys. Rev. Lett. 21, 244 (1968).

[51] L. M. Barkov et al., Nucl. Phys. B 256, 365 (1985).

[52] M. Hoferichter et al., Eur. Phys. J. A 52, 331 (2016).

[53] Z. Epstein, G. Paz, and J. Roy, Phys. Rev. D 90, 074027 (2014).

[54] C. Alexandrou, M. Constantinou, K. Hadjiyiannakou, K. Jansen, Ch. Kallidonis, G. Koutsou, and A. Vaquero Aviles-Casco, Phys. Rev. D 96, 034503 (2017).

[55] M. A. Belushkin, H.-W. Hammer, and Ulf-G. Meißner, Phys. Lett. B 633, 507 (2006).

[56] C. Patrignani et al. (Particle Data Group), Chin. Phys. C 40, 100001 (2016); and 2017 update.

[57] G. A. Miller, Annu. Rev. Nucl. Part. Sci. 60, 1 (2010).

[58] J. C. Bernauer, Doctoral dissertation, Mainz University, 2010 (unpublished).

[59] M. O. Distler, J. C. Bernauer, and T. Walcher, Phys. Lett. B 696, 343 (2011).

[60] J. M. Alarcón, D. Higinbotham, and C. Weiss (unpublished).

[61] C. Granados and C. Weiss, J. High Energy Phys. 07 (2015) 170.

[62] C. Granados and C. Weiss, Phys. Rev. C 92, 025206 (2015).

[63] C. Granados and C. Weiss, J. High Energy Phys. 06 (2016) 075.

[64] T. Ledwig, J. Martin-Camalich, V. Pascalutsa, and M. Vanderhaeghen, Phys. Rev. D 85, 034013 (2012).

[65] T. Ledwig, V. Pascalutsa, and M. Vanderhaeghen, Phys. Rev. D 82, 091301 (2010).

[66] M. Mai, B. Hu, M. Doring, A. Pilloni, and A. Szczepaniak, Eur. Phys. J. A 53, 177 (2017).

[67] R. A. Briceno, M. T. Hansen, and S. R. Sharpe, EPJ Web Conf. 137, 05004 (2017).

[68] J. M. Alarcón, J. M. Camalich, and J. A. Oller, Phys. Rev. D 85, 051503 (2012).

[69] J. M. Alarcón, L. S. Geng, J. M. Camalich, and J. A. Oller, Phys. Lett. B 730, 342 (2014).

[70] J. M. Alarcón, V. Lensky, and V. Pascalutsa, Eur. Phys. J. C 74, 2852 (2014). 